\title{
Use of Orthotropic Plate Model Dynamics to Design for Endurance of Heat Transfer Plates
}

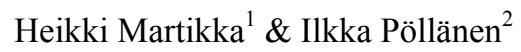 \\ ${ }^{1}$ Himtech Oy, Engineering, Ollintie 4, Joutseno, Finland \\ ${ }^{2}$ SAV Oy, Savonkatu 21, Kouvola, Finland \\ Correspondence: Heikki Martikka, Himtech Oy, Engineering, Ollintie 4, Joutseno FIN-54100, Finland. Tel: \\ 358-408-393-167. E-mail: heikki.martikka@pp.inet.fi
}

Received: March 26, $2013 \quad$ Accepted: June 5, $2013 \quad$ Online Published: July 15, 2013
doi:10.5539/mer.v3n2p28

This research is financed by Himtech $\mathrm{Oy}$ and SAV Oy

\begin{abstract}
In this study results are presented of a study of applying surrogate plate models for dynamic design of structurally orthotropic heat transfer plates. The plates are assembled at fixed supports at upper and lower ends. One plate consists of parallel tubes joined by intermediate plates to each other.

They are loaded by dynamically varying hot flue gas flow and pressure loads and by random soot mass impact loads due to operation conditions. These cause fatiguing stresses leading to fracturing and shutdowns and explosions. The goal is to obtain a predictive design model for increasing the reliable useful operating time. Several available methods are used in a larger study, matrix based, composite structure based and continuous beam based methods. In this study the orthotropic plate surrogate material approach is chosen. The dynamic behaviors are measured .The surrogate model predictions and measurements agree well. The predictions of the homogeneous surrogate orthotropic plate agree well with FEM model predictions. FEM modeling of complex structures made of orthotropic plates can be made cost effectively with reasonable accuracy using this surrogate plate approach.
\end{abstract}

Keywords: beams, orthotropic plates, dynamics, optimum design, FEM, soot, recovery boilers

\section{Introduction}

\subsection{Background and Motivation}

Orthotropic and composite plates and shells are important basic components of the technological infrastructures. They are used extensively in static and mobile structures. Now objects of study are recovery boilers and their heat transfer plates. They are assembled in parallel vertically and stiffly supported at upper and lower ends. Their endurance is essential for the whole process.

Since the plates are orthotropic and complex in geometry they are difficult and costly to calculate in detail even with best FEM. Therefore cost-effective surrogate models are needed which are fast, easy to use and accurate enough. Now orthotropic plate models are applied to construct surrogate plate FEM model to simulate accurately enough the dynamic behavior of the actual plate.

Orthotropic plate composite theory and dynamics are discussed by (Swanson, 1997), (Barbero, 1999) and (Agarwal \& Broutman, 1990). General plate theory is discussed by (Szilard, 1975). The optimum fuzzy design is used and discussed by (Martikka \& Pöllänen, 2009). The applicability and utility of this method is shown in the design of suspension bridges (Martikka \& Taitokari, 2013). This method is based on results of (Diaz, 1988). Structural analysis of plates, beams and shells by (Case et al., 1993), (Boresi, 1993) and (Ventsel \& Krauthammer, 2001) can be used. Flow induced vibration and flutter are important in parallel plates under process conditions as discussed by (Blevins, 1990) and by (Dimarogonas, 1992). Since consequences of boiler failures are serious they should be designed with the reliability based design approach, as discussed by (Dhillon et al., 1981) and (Leitch, 1988). In this study the advanced FEM program NX Nastran is applied. Basic engineering data is essential in calculations as by (Dubbel Handbook, 1994). Surrogate beam models for 
dynamic design of a complex heat transfer plates are useful in design of recovery boilers as discussed by (Martikka, Pöllänen, \& Simonen, 2006).

Extensive literature surveys made by a recovery boiler manufacturer and engineering design companies showed that the published focus has been narrowly on varying external process control variables within restricted limits like, pressure, temperatures, and flows in and out with the aim of minimizing soot layer thickness without destroying the boiler. One example is the rapport by (Jamee, Araned, \& Luks, 1998). It explains techniques of extending the recovery boiler runtime through temperation of steam at soot blower. But there are no studies focused on the internal dynamics and endurance of the boiler and new innovations for removing the soot like the present study. The aim of this study is to optimize energy efficiency by controlling the endurance of internal components and minimizing pluggages and soot layer thickness.

\subsection{Goals and Outline}

The goals and outline of this work are described.

The overall goal is to promote the reliable, safe and cost-effective operation of the recovery boilers. Main hindrances are soot accumulation to heat transfer plates causing energy losses. Other risks are the process flow and soot cleansing flow induced fatiguing vibrations. But modeling of a collective of interacting complex plates is prohibitive for even the best FEM. Thus a surrogate easy to use FEM plate model is needed.

(1) The first goal is development of methodology for making a surrogate plate models for an orthotropic plate which is accurate enough for describing the dynamic behavior of the plates.

(2) The second goal is verification of the predictions of the surrogate FEM model of the orthotropic plate by comparing with experimental measurements and with accurate FEM model predictions.

(3) The third goal is to develop a physical soot growth and removal model which can be used to design new means of its removal and retarding its growth. These results are not shown in this paper.

The outline in this work:

First the dynamic behavior model of the orthotropic plates is reviewed. Then the derivations of the orthotropic plate surrogate parameters are presented. It is based on actual steel plate geometry.

Next these surrogate model parameters are used in a plate FEM model. These results are compared with the experimental modal analysis results of test plates. Then the utility of the surrogate models is extended to get a quick stress strain and fatigue endurance estimates.

\section{The Studied Structure}

The objective in this section is to describe the overall structure of a recovery boiler. The specific objects of this study are the orthotropic heat transfer plates. Their cost effective function is of paramount importance for the whole system. The major problems are heat efficiency loss due to soot accumulation and fatiguing vibrations.

\subsection{Geometry and Loading}

Sketches of the structure and its loads are shown in Figures 1(a) and 1(b). One plate may be modeled as a stiffly supported beam. Figure 1(c) shows a typical pluggage prior to soot blowing (Jamee, Araned, \& Luks, 1998). 


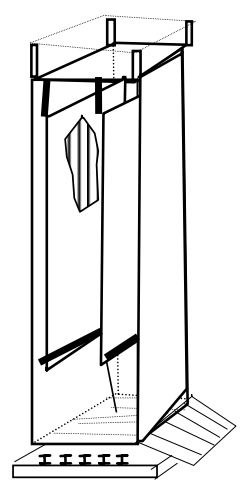

(a)

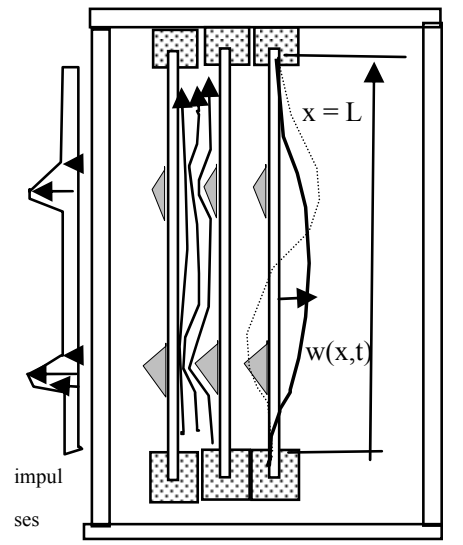

(b)

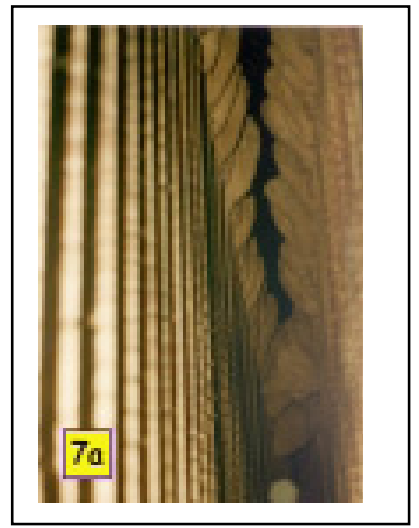

(c)

Figure 1. Sketch of main structure of a typical recovery boiler: a) Main dimensions; b) Flow pressure variations due to soot clogging due to pressure load peaks on the plates exciting deleterious vibrations;

c) Typical pluggage prior to soot blowing (Jamee, Araned, \& Luks, 1998)

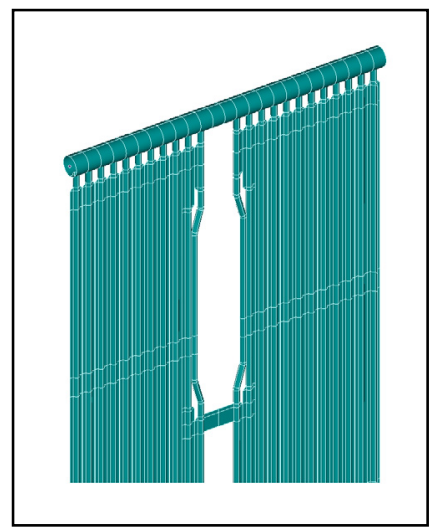

(a)

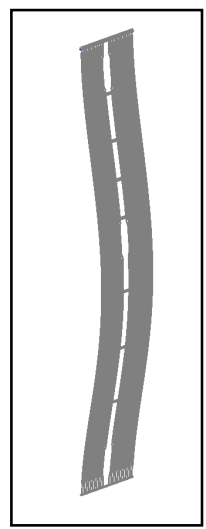

(b)

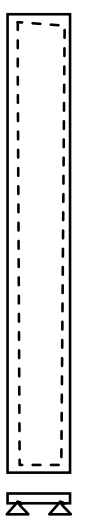

(c)

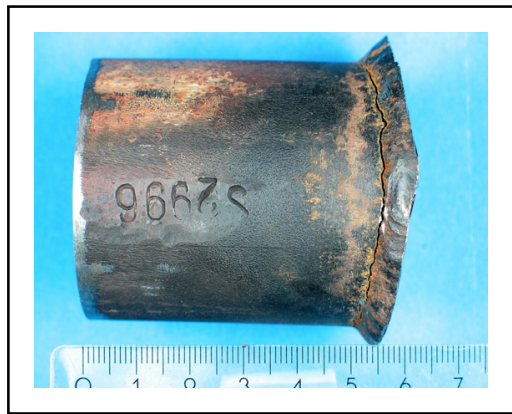

(d)

Figure 2. Heat transfer plates: a) FEM modeling of whole plate with 1.15 million elements

The length is $23 \mathrm{~m}$ and total width $2.8 \mathrm{~m}$ with $0.28 \mathrm{~m}$ gap with stiff supports at at upper and lower ends and free sides; b) The first modal form by FEM had eigenfrequency $0.545 \mathrm{~Hz}$; c) The orthotropic analytic plate with length $23 \mathrm{~m}$, width $5 \mathrm{~m}$ and with all edges freely supported gave higher frequency $f_{\text {anal }}=2.86 \mathrm{~Hz}$ due to stiffer free-support conditions at all edges; d) Typical tube weld fracture

\subsection{Structure of the Orthotropic Plate}

In Figures 2(a), (b) and (c) the detailed FEM model is shown. In Figure 2(d) a typical weld fracture is shown.

Plate models are shown in Figure 3. The main goal is to get a surrogate model for the plate which can be used to predict roughly the same lowest eigenfrequency as by FEM model. The second goal is to get estimates for stresses and deflections. Using the surrogate model it is possible to model cost-effectively large and geometrically complex structures fast and with reasonable accuracy.

\section{Dynamic Behavior of the Orthotropic Plate}

The objective of this section is to give a detailed enough description of the dynamic behavior of orthotropic plates. Basic definitions are shown in Figure 3. 

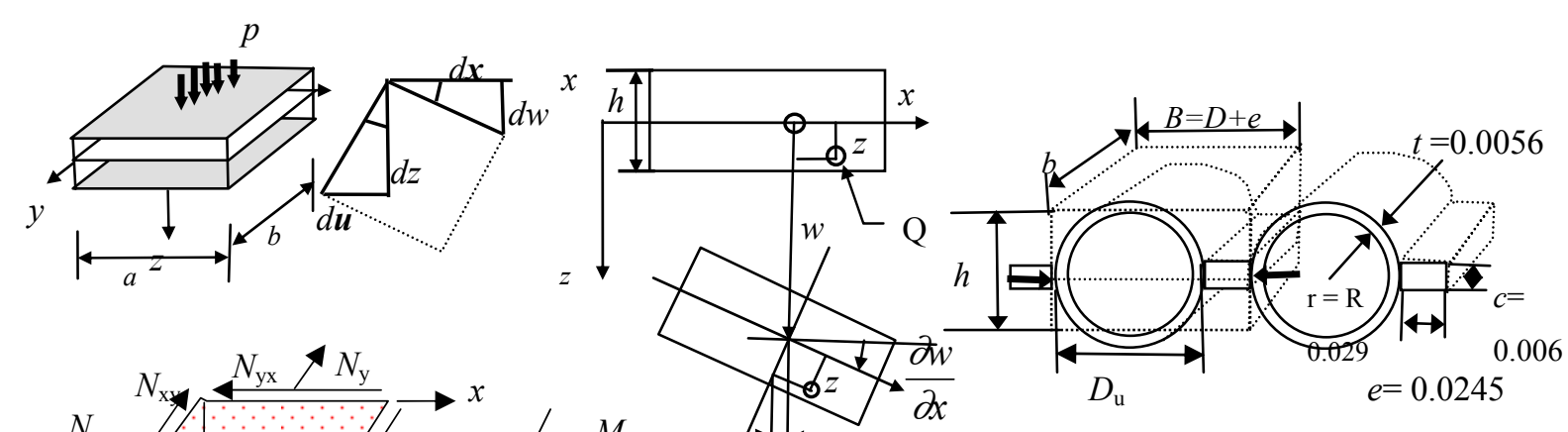

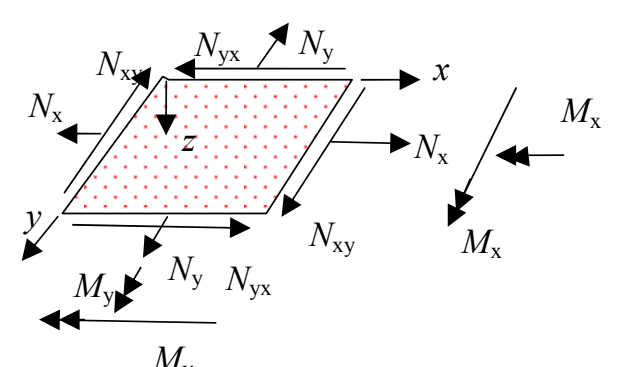

(a)

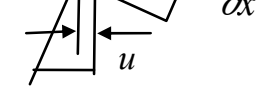

$$
\frac{\partial u}{\partial z}
$$

Figure 3. Plate models: a) Plate deformation and stress resultant definitions;

b) The basic structure and dimensions

The dimensions of the cross section in Figure $3 \mathrm{~b}$ ) are

$$
D_{u}=0.0635, \quad t=0.0056, \quad D=D_{u}-t=0.0579, \quad e=0.0245, c=0.006
$$

The governing equation of the plate is by (Swanson, 1997)

$$
\frac{\partial^{2} M_{\mathrm{x}}}{\partial x^{2}}+2 \frac{\partial^{2} M_{\mathrm{xy}}}{\partial x \partial y}+\frac{\partial^{2} M_{\mathrm{y}}}{\partial y^{2}}=-p(x, y),\left[\begin{array}{c}
\frac{\partial^{2}}{\partial x^{2}} \\
\frac{\partial^{2}}{\partial x^{2}} \\
2 \frac{\partial^{2}}{\partial x \partial y}
\end{array}\right]^{T}\left[\begin{array}{c}
M_{x} \\
M_{y} \\
M_{x y}
\end{array}\right]=-p
$$

Here the $M_{\mathrm{ik}}$ are moments and $p$ is pressure as illustrated in Figure 3. Next the moment vector is expressed as function of the deflection $w$ as illustrated in Figure 3.

If neutral plane strains are zero then the relationships between moment resultants, curvatures and stiffness properties are

$$
\left[\begin{array}{l}
M_{x} \\
M_{y} \\
M_{x y}
\end{array}\right]=\left[\begin{array}{lll}
D_{11} & D_{12} & D_{16} \\
D_{12} & D_{22} & D_{26} \\
D_{16} & D_{26} & D_{66}
\end{array}\right]\left[\begin{array}{c}
\kappa_{\mathrm{x}} \\
\kappa_{\mathrm{y}} \\
\kappa_{\mathrm{xy}}
\end{array}\right] \quad, \quad \mathbf{M}=\mathbf{D} \kappa \quad,\left[\begin{array}{c}
\kappa_{\mathrm{x}} \\
\kappa_{\mathrm{y}} \\
\kappa_{\mathrm{xy}}
\end{array}\right]=\left[\begin{array}{c}
-w_{\mathrm{xx}} \\
-w_{\mathrm{yy}} \\
-2 w_{\mathrm{xy}}
\end{array}\right]
$$

Substituting here the elements of $\mathbf{D}$ gives (Swanson, 1997)

$$
\left[\begin{array}{l}
M_{x} \\
M_{y} \\
M_{x y}
\end{array}\right]=\frac{h^{3}}{12}\left[\begin{array}{ccc}
Q_{11} & Q_{12} & 0 \\
Q_{12} & Q_{22} & 0 \\
0 & 0 & Q_{66}
\end{array}\right]\left[\begin{array}{c}
\kappa_{\mathrm{x}} \\
\kappa_{\mathrm{y}} \\
\kappa_{\mathrm{xy}}
\end{array}\right], \quad \mathbf{M}=\frac{h^{3}}{12} \mathbf{Q} \kappa \quad, \quad \mathbf{D}=\frac{h^{3}}{12} \mathbf{Q}
$$

The stress strain model of Hooke's law for an orthotropic lamina in principal material co-ordinates LT is

$$
\left[\begin{array}{c}
\sigma_{11} \\
\sigma_{22} \\
\tau_{12}
\end{array}\right]=\left[\begin{array}{ccc}
\frac{E_{\mathrm{L}}}{1-v_{\mathrm{LT}} v_{\mathrm{TL}}} & \frac{v_{\mathrm{LT}} E_{\mathrm{T}}}{1-v_{\mathrm{LT}} v_{\mathrm{TL}}} & 0 \\
\frac{v_{\mathrm{LT}} E_{\mathrm{T}}}{1-v_{\mathrm{LT}} v_{\mathrm{TL}}} & \frac{E_{\mathrm{T}}}{1-v_{\mathrm{LT}} v_{\mathrm{TL}}} & 0 \\
0 & 0 & G_{\mathrm{LT}}
\end{array}\right]\left[\begin{array}{c}
\varepsilon_{11} \\
\varepsilon_{22} \\
\gamma_{12}
\end{array}\right], \frac{v_{21}}{E_{2}}=\frac{v_{12}}{E_{1}} \quad,\{\sigma\}=\mathbf{D}\{\varepsilon\}
$$

Here the principal material directions are denoted by: $\mathrm{L}=$ Longitudinal, $\mathrm{T}=\mathrm{Transverse}$ direction 
Substituting Equation (2) to Equation (1) one obtains

$$
\left[\begin{array}{c}
\frac{\partial^{2}}{\partial x^{2}} \\
\frac{\partial^{2}}{\partial x^{2}} \\
2 \frac{\partial^{2}}{\partial x \partial y}
\end{array}\right]^{T}\left\{\left[\begin{array}{lll}
D_{11} & D_{12} & D_{16} \\
D_{12} & D_{22} & D_{26} \\
D_{16} & D_{26} & D_{66}
\end{array}\right]\left[\begin{array}{l}
-w_{\mathrm{xx}} \\
-w_{\mathrm{yy}} \\
-2 w_{\mathrm{xy}}
\end{array}\right]\right\}=-p
$$

Next performing the first matrix operation gives

$$
\left[\begin{array}{c}
\frac{\partial^{2}}{\partial x^{2}} \\
\frac{\partial^{2}}{\partial y^{2}} \\
2 \frac{\partial^{2}}{\partial x \partial y}
\end{array}\right]^{T}\left[\begin{array}{l}
D_{11} w_{\mathrm{xx}}+D_{12} w_{\mathrm{yy}}+D_{16} 2 w_{\mathrm{xy}} \\
D_{12} w_{\mathrm{xx}}+D_{22} w_{\mathrm{yy}}+D_{26} 2 w_{\mathrm{xy}} \\
D_{16} w_{\mathrm{xx}}+D_{26} w_{\mathrm{yy}}+D_{66} 2 w_{\mathrm{xy}}
\end{array}\right]=-p
$$

Now some approximations can be used to simplify the results without sacrificing accuracy. The effect of shear deformations on bending deformations are assumed to be so small that they may be neglected

$$
D_{16}=0, D_{26}=0
$$

The equation of static deflection is now simplified. By adding the dynamic inertia volume force term, the equation of motion of the plate becomes

$$
\begin{aligned}
& \left(D_{11} w_{\mathrm{xxxx}}+D_{12} w_{\mathrm{yyxx}}+0 \cdot 2 w_{\mathrm{xyxx}}\right)+\left(D_{12} w_{\mathrm{xxyy}}+D_{22} w_{\mathrm{yyyy}}+0 \cdot 2 w_{\mathrm{xyyy}}\right)+ \\
& +2\left(0 \cdot w_{\mathrm{xxxy}}+0 \cdot w_{\mathrm{yyxy}}+D_{66} 2 w_{\mathrm{xyxy}}\right)=p-\rho_{\mathrm{a}} \ddot{w}
\end{aligned}
$$

Eigenfrequencies are calculated with the following equation (Swanson, 1997)

$$
D_{11} w_{\mathrm{xxxx}}+2\left(D_{12}+2 D_{66}\right) w_{\mathrm{yyxx}}+D_{22} w_{\text {yyyy }}=p-\rho_{\mathrm{a}} \ddot{w}
$$

Here $\rho_{\mathrm{a}}$ is mass per unit area.

Trial solution for the static deflection equation under static distributed pressure $p$ is

$$
w(x, y)=\sum_{m=1}^{\infty} \sum_{n=1}^{\infty} C_{m n} \sin (\alpha \pi x) \sin (\beta \pi y) \quad \alpha=\frac{m}{a} \quad, \beta=\frac{n}{b}
$$

Dynamic deflection is now product some of eigenmode shape functions and eigentime functions

$$
w(x, y, t)=\sum W(x) q(t)=\sum W_{m n}(x) \cos \omega_{m n} t
$$

Now a cosine time function may be chosen without loss of generality

$$
w(x, y, t)=\sum_{m=1}^{\infty} \sum_{n=1}^{\infty} G_{\mathrm{mn}} \sin (\alpha \pi x) \sin (\beta \pi y) \cos \omega_{\mathrm{mn}} t
$$

Derivatives are needed

$$
\begin{aligned}
& w_{\mathrm{xxxx}}(x, y, t)=\sum_{m=1}^{\infty} \sum_{n=1}^{\infty} G_{\mathrm{mn}}(\alpha \pi)^{4} \sin (\alpha \pi x) \sin (\beta \pi y) \cos \omega_{\mathrm{mn}} t \\
& \rho_{a} \ddot{w}(x, y, t)=\sum_{m=1}^{\infty} \sum_{n=1}^{\infty} G_{\mathrm{mn}} \sin (\alpha \pi x) \sin (\beta \pi y)\left(\omega_{\mathrm{mn}}\right)^{2} \cos \omega_{\mathrm{mn}} t
\end{aligned}
$$

Eigenfrequencies are obtained from equation of free vibrations by setting load to zero, $\quad p=0$.

Substitution of the freely supported solution to the equation of motion 


$$
D_{11} w_{\mathrm{xxxx}}+2\left(D_{12}+2 D_{66}\right) w_{\mathrm{yyxx}}+D_{22} w_{\mathrm{yyyy}}+\rho_{\mathrm{a}} \ddot{w}=0
$$

Gives

$$
\left[\sum_{m=1}^{\infty} \sum_{n=1}^{\infty} W_{\mathrm{mn}}\right] \pi^{4}\left[D_{11} \alpha^{4}+2\left(D_{12}+2 D_{66}\right) \alpha^{2} \beta^{2}+D_{22} \beta^{4}-\frac{\rho_{\mathrm{a}}}{\pi^{4}} \omega_{\mathrm{mn}}{ }^{2}\right]=0
$$

Each coefficient in the terms of the sum must vanish. The eigenfrequencies are obtained as

$$
\omega_{\mathrm{mn}}=\frac{\pi^{2}}{\sqrt{\rho_{\mathrm{a}}}}\left[D_{11} \alpha^{4}+2\left(D_{12}+2 D_{66}\right) \alpha^{2} \beta^{2}+D_{22} \beta^{4}\right]^{\frac{1}{2}} \quad, \quad \alpha=\frac{m}{a} \quad \beta=\frac{n}{b}
$$

Mass per unit area is obtained by dividing the mass by its mid plane area $A$,

$$
\rho_{\mathrm{a}}=\frac{\rho_{\text {steel }} V}{A^{\prime}}=\frac{\rho_{\text {steel }}(2 \pi r t+c e) b}{b \cdot(2 r+e)}=\rho_{\text {steel }} \frac{\left(2 \pi t+c \frac{e}{r}\right)}{2+\frac{e}{r}}=111 \frac{\mathrm{kg}}{\mathrm{m}^{2}}
$$

\section{Orthotropic Plate Parameters}

The first objective in this section is to develop a methodology for formulating surrogate plate models to simulate accurately enough the dynamic and static behavior of an orthotropic plate of the recovery boiler. Orthotropic plate parameters are now needed for the dynamic modeling.

\subsection{Axial Loading and Bending Loading Surrogate Elastic Models for the Axial Direction}

The objective is that the surrogate plate would approximate the behavior of the actual plate in axial and bending loading. Here $E$ is elastic modulus and $A$ is cross sectional area transverse to the tension direction of the plate. Two models can be used to estimate the surrogate axial elastic modulus.

Model A: Equality of axial stiffnesses EA gives an estimate for $E_{1, \text { tension }}$

$$
\begin{aligned}
& \left(E_{1, \mathrm{t}} A\right)_{\text {surrogate }}=(E A)_{\text {steel }} \Rightarrow E_{1, \text { tension }}=\frac{A_{\text {steel }}}{A_{1, \text { surrogate }}} E \Rightarrow \frac{\pi D t+e c}{h L} E \Rightarrow \\
& \frac{E_{1, \text { tension }}}{E}=\frac{e c}{L h}\left(2 \pi \frac{r t}{e c}+1\right)
\end{aligned}
$$

Model B: Equality of bending stiffnesses EI gives an estimate for $E_{1, \text { bending }}$

$$
\left(E_{1, \mathrm{~b}} I\right)_{\text {surrogate }}=(E I)_{\text {steel }} \Rightarrow E_{1, \text { bending }}=\frac{12}{L h^{3}}\left[\pi r^{3} t+\frac{e c^{3}}{12}\right] \bullet E
$$

These results for the surrogate moduli by the Model A and B may be summarized as

$$
\begin{aligned}
& \frac{E_{1, \mathrm{t}}}{E}=\left(\frac{e c}{L h}\right)\left(2 \pi \frac{r t}{e c}+1\right) \\
& \frac{E_{1, \mathrm{~b}}}{E}=\left(\frac{e c}{L h}\right)\left(12 \pi \frac{r t}{e c}\left(\frac{r}{h}\right)^{2}+\left(\frac{c}{h}\right)^{2}\right)
\end{aligned}
$$

The average is used as a trade-off compromise

$$
E_{1}=0.5\left(E_{1, \mathrm{t}}+E_{1, \mathrm{~b}}\right)=0.5(4280+4460)=4370 \mathrm{MPa}
$$

\subsection{Transverse Tension and Bending Elastic Moduli}

Several models are used to estimate them. The actual plates have the steel material modulus of elasticity. But in surrogate modeling the transverse bending elasticity is described by a fictive own modulus and in transverse tension with another. Only one elastic modulus must be chosen. Thus a trade-off compromise is needed to choose only one optimal transverse elastic modulus. 


\subsubsection{Same Bending Stiffness Model}

In Figure 4 the bending force $F_{2}=Q_{2} \cdot b c$ acts at the end of the flange with an effective lever. This lever is a design variable for obtain optimized material properties. Now transverse bending is considered. The assumption is that by equating the bending stiffness for the surrogate plate and for the steel plate one will get a reasonable estimate for the transverse elastic modulus in bending

$$
\begin{aligned}
k_{2, \text { surrogate }}=k_{2, \text { steel }} & =\frac{f}{F}=\frac{\text { deflection.at.force }}{\text { force }} \\
{\left[\frac{E_{2, b}}{3 L_{2}{ }^{3}} \frac{b h^{3}}{12}\right]_{\text {surrogate }} } & =\left[\frac{E}{3 e^{3}} \frac{b c^{3}}{12}\right]_{\text {steel }}
\end{aligned}
$$

Thus the following equation can be used to get an approximation for the elastic modulus under bending

$$
E_{2, \mathrm{~b}}=E\left(\frac{L_{2}}{e}\right)^{3}\left(\frac{c}{h}\right)^{3}=E\left(\frac{L_{2}}{24.5}\right)^{3}\left(\frac{6}{65}\right)^{3}
$$

Here $L_{2}$ is a lever parameter of the model which can be tuned to obtain optimal model accuracy, Figure $4 \mathrm{~b}$ ).

- If $L_{2}=e=L_{2, \min }$ then $E_{2 \mathrm{~b}}=200 \mathrm{MPa}$, this is the minimal estimate

- if $L_{2}=1 / 2 D+e=L_{2 \cdot \max }$ then $E_{2, \mathrm{~b}}=2100 \mathrm{MPa}$, this is the maximal estimate

Thus a reasonable value can be assumed to be in the range $200<E_{2 \mathrm{~b}}<2100 \mathrm{MPa}$.

A trade-off compromise value is $E_{2}=800 \mathrm{MPa}$.

\subsubsection{Use of the Compressed Circular Spring Model to Obtain Transverse Elasticity}

In transverse loading the tube may be modeled as circular ring of diameter $D$ and thickness $t$ and some length $b$ as shown in Figure 4a). The force loaded circular spring model is taken from (Ylinen, 1970)

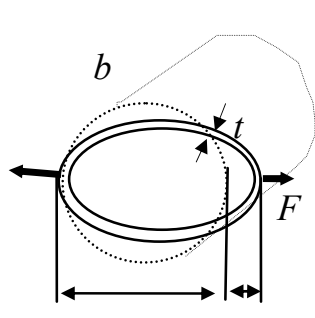

$D$

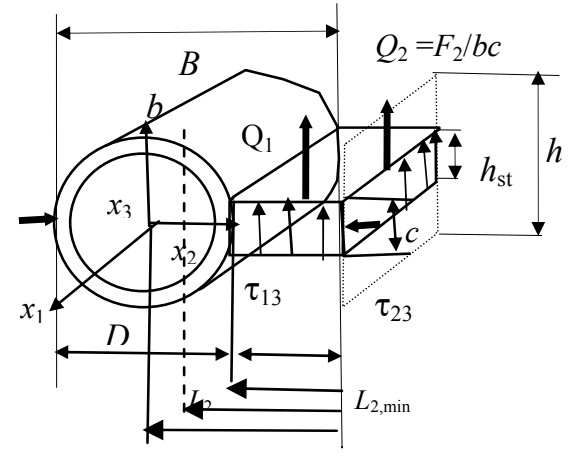

(b)

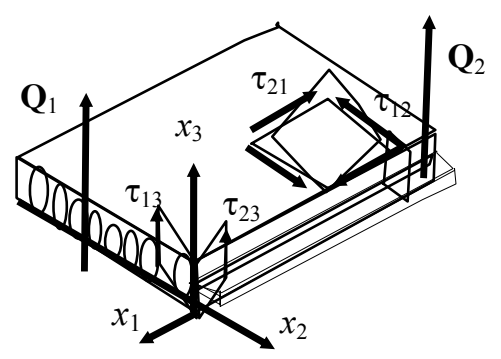

(c)

Figure 4. Basic geometry and loading: a) Compressed ring model to model the transverse stiffness; b) Shear and transverse compressive forces acting on a module of structure; c) Orthotropic plate model

Change in diameter $D$ of the ring by force $F$ is the spring deflection

$$
\Delta D=0.149 \frac{r^{3}}{E I_{1}} F=f=\frac{1}{k_{\text {tube }}} F \quad, \quad I_{1}=I=\frac{1}{12} b t^{3} \quad, \quad r=1 / 2 D
$$

From this one can solve the spring stiffness of the tube as

$$
k_{\text {tube }}=\frac{1}{z} E b\left(\frac{t}{r}\right)^{3}, \quad z=0.149 \cdot 12=1.788
$$


An estimate for the transverse tension-compression modulus $E_{2 \mathrm{t}}$ may be obtained from the equivalence of the transverse compression spring stiffness of the plate and the surrogate plate model

$$
k_{2, \text { surrogate }}=\frac{1}{\frac{1}{k_{2, \text { tube }}}+\frac{1}{k_{2, \text { plate }}}} \approx k_{2, \text { tube }}=\frac{E_{2 t} A_{2}}{L}=\frac{1}{z} E b\left(\frac{t}{r}\right)^{3}, A_{2}=b h
$$

Assuming surrogate thickness $h=65 \mathrm{~mm}$ gives

$$
E_{2, t}=\frac{1}{z}\left(\frac{t}{r}\right)^{3} \frac{L}{h} E=\frac{1}{1.788}\left(\frac{5.6}{29}\right)^{3} \frac{88}{65} E=1150 M P a
$$

Two estimates are obtained for the transverse modulus of elasticity

- Equating the two tension spring stiffness models gave an estimate $E_{2, \mathrm{t}}=1150$.

- Equating the two transverse bending stiffness models gave a range $E_{2, \mathrm{~b}}=200 \ldots 2100$.

The compromise value $E_{2}=800$ is chosen.

\subsection{Estimation of Surrogate Shear Moduli}

Shear modulus $G$ for isotropic steel is a material property of the isotropic elastic solid

$$
G=\frac{E_{1}}{2\left(1+v_{12}\right)} \Rightarrow \frac{210000}{2(1+0.3)}=80770 \mathrm{MPa}=G_{12}=G_{21}
$$

Now the orthotropic plate the shear modulus $G_{12}$ is often approximated as depending on the two principal elastic moduli. The model by (Ventsel, Krauthammer, 2001) is used. The effect of Poisson's ratio is neglected for simplicity

$$
\begin{aligned}
& E_{1}=4370, \quad E_{2}=800 \\
& G_{12} \approx 0.5\left(E_{1} \cdot E_{2}\right)^{\frac{1}{2}} \approx 3000=G_{\text {ventsel }}
\end{aligned}
$$

The moduli $G_{13}$ and $G_{23}$ are needed for 3D modeling with the surrogate material but not for 2D modeling.

The same shear force is transmitted through the surrogate plate cross section and also through the corresponding cross section of the steel structure. From this force balance one obtains the surrogate modulus

$$
G_{23}=G \frac{c}{h}=80770 \frac{0.006}{0.065}=7455
$$

Equating the shear forces and shear angles in the transverse to the main direction gives

$$
\begin{aligned}
& F_{\text {shear,surrogate }}=G_{13} h L \bullet \gamma_{13}=F_{\text {shear,steel }}=G_{13, \text { tube }} \pi D t \bullet \gamma_{13} \\
& G_{13} h L=G_{13, \text { tube }} \pi D t
\end{aligned}
$$

Thus the shear modulus $G_{13}$ estimate is obtained as follows

$$
G_{13}=G \frac{\pi t}{h} \cdot \frac{1}{1+\frac{e}{D}} \approx G \frac{\pi t}{h}=80770 \frac{\pi 0.0056}{0.065}=21900
$$

\section{Results}

The results of this work show that the initial goals were satisfactorily well achieved.

(1) The first result is a methodology for formulating a surrogate plate model for an orthotropic plate of the recovery boiler.

(2) The second result is the verification of the surrogate FEM model of the orthotropic plate by comparing with experimental measurements and with the accurate FEM model predictions.

(3) The third result in this project is a physical soot growth and removal model which can be used to design new methods of its removal and retarding its growths. Now it is outside the scope of this paper. 


\subsection{Results of Parameters for FEM Modeling}

First the parameters of the surrogate model are summarized and some results obtained by using them.

Table 1. Results of varying the surrogate plate thickness variables to get optimal elastic moduli (MPa) for use in FEM

\begin{tabular}{ll}
\hline$h$,surrogate plate thickness & $\boldsymbol{h}=\mathbf{6 5} \mathbf{~ m m}$ \\
\hline$E_{1, \mathrm{~b}} \quad \mathrm{~b}=$ bending & 44600 \\
$E_{1, \mathrm{t}} \quad \mathrm{t}=$ tension & 42800 \\
$\boldsymbol{E}_{\mathbf{1}}$, used in axial direction & $\mathbf{4 3 7 0 0}$ \\
$E_{2 \mathrm{~b}}$, transverse bending estimate range & $200 \ldots 2100$ \\
$E_{2 \mathrm{t}}$, transverse tension estimate & 1150 \\
$\boldsymbol{E}_{\mathbf{2}}$, used in transverse compromise & $\mathbf{8 0 0}$ \\
$G$, steel & 80770 \\
$\boldsymbol{G}_{\mathbf{1 3}}$, used in 3D models & $\mathbf{2 1 9 0 0}$ \\
$\boldsymbol{G}_{\mathbf{2 3}}$, used in 3D models & $\mathbf{7 4 6 0}$ \\
$\boldsymbol{G}_{\mathbf{v e n t s e l}}=\boldsymbol{G}_{\mathbf{1 2}}$ used as compromise & $\mathbf{3 0 0 0}$ \\
$\boldsymbol{v}_{\mathbf{2 1}}, \boldsymbol{v}_{\mathbf{1 2}}$, Poisson's ratios for plane models & $\mathbf{0 . 3 , 0 . 0 5 5}$ \\
$\boldsymbol{v}_{\mathbf{3 1}}, \boldsymbol{v}_{\mathbf{1 3}}$, used in 3D models & $\mathbf{0 . 3 , 0 . 3}$ \\
\hline
\end{tabular}

The $\mathbf{D}$ matrix is obtained as follows from Equations (2), (3) and (4)

$$
\begin{aligned}
& D=\left[\begin{array}{lll}
D_{11} & D_{12} & D_{16} \\
D_{12} & D_{22} & D_{26} \\
D_{16} & D_{26} & D_{66}
\end{array}\right]=\frac{h^{3}}{12}\left[\begin{array}{ccc}
Q_{11} & Q_{12} & 0 \\
Q_{12} & Q_{22} & 0 \\
0 & 0 & Q_{66}
\end{array}\right]=\frac{h^{3}}{12}\left[\begin{array}{ccc}
E_{1} & v_{12} E_{2} & 0 \\
v_{12} E_{2} & E_{2} & 0 \\
0 & 0 & G_{12}
\end{array}\right] \\
& \mathbf{D}=\frac{h^{3}}{12} \mathbf{Q} \quad, \quad v_{12}=0.3 \quad, \quad G_{12}=\frac{1}{2}\left(E_{1} \cdot E_{2}\right)^{0.5} \quad, \quad u=\frac{h^{3}}{12}
\end{aligned}
$$

Comparison of predictions for the lowest eigenfrequency by three methods is shown in Table 2.

Table 2. Analytical and FEM surrogate plate eigenfrequencies using the data in Table 1 with surrogate thickness $h=0.065 \mathrm{~m}$. The lowest eigenfrequency is presented, $\mathrm{m}=1$ is number of periods in main 1-direction, $\mathrm{n}=1$ is number of periods in transverse direction

\begin{tabular}{lccccc}
\hline $\begin{array}{c}\text { side of } \\
\text { plate }\end{array}$ & $\begin{array}{c}\text { side of } \\
\text { plate }\end{array}$ & specimen & FEM & Analytical & FEM \\
$a(\mathrm{~m})$ & $b(\mathrm{~m})$ & & $\begin{array}{c}\text { Surrogate plate model free } \\
\text { support-free support }\end{array}$ & $\begin{array}{c}\text { orthotropic plate model free } \\
\text { support }- \text { free support }\end{array}$ & $\begin{array}{c}\text { Large detailed plate } \\
\text { model }\end{array}$ \\
\hline$a$ & $b$ & & $f_{11}(\mathrm{~Hz})$ & $f_{\mathrm{mn}}(\mathrm{a}, \mathrm{b})(\mathrm{Hz})$ & $f_{\mathrm{mn}}(\mathrm{a}, \mathrm{b})(\mathrm{Hz})$ \\
\hline 1.314 & 0.803 & test plate & $\mathbf{1 1 4}$ & $\mathbf{1 1 8}$ & - \\
$23=L$ & $2.8=b$ & FEM size & - & $\mathbf{2 . 8 6}$ & $\mathbf{0 . 5 4 5}$ \\
\hline
\end{tabular}




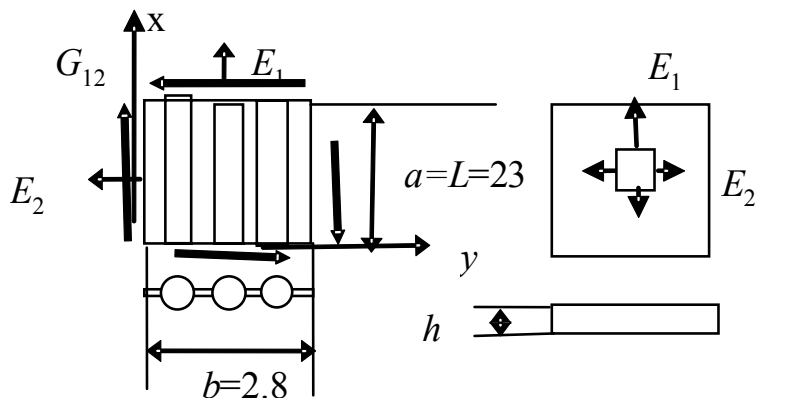

(a)

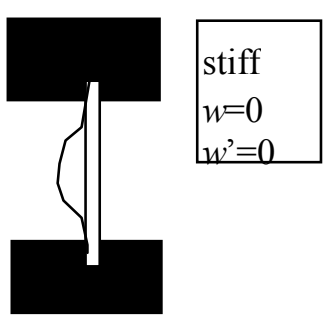

(c)

Figure 5. Sketches of plates: a) Heat transfer plate main dimensions; b) Surrogate orthotropic plate with same mid plane dimensions $a$ and $b$ and with even thickness $h$; c) Support conditions for a stiff-stiff beam

\subsection{FEM Results for the Test Plate}

The dynamic behavior of the test plate is analyzed using FEM model with constant thickness plate elements of surrogate material and two boundary conditions. The objective is a verification of the validity and accuracy of the surrogate FEM model of the orthotropic plate by comparing its predictions with experimental measurements and with accurate FEM model predictions.

\subsubsection{Frequencies of the Test Plate with all Edges Fully Free Using Measurements and FEM Surrogate Model}

Measurement of frequencies was made by (Talvio, 2009) using a modal analysis instrument with three positions for the accelerometer. The plate was supported by a rod loosely fitted inside the upper tube. It was nearly free-free supported along upper edge. The mode shapes were not measured.

These measurements can be interpreted using the surrogate plate FEM modal analysis results in Figures 6 and 7. It had the same main dimensions and areal mass density as the steel test plate. The results are shown in Table 3. Comparison shows that with high probability the measured mode shapes were as predicted by the FEM results.

Table 3. The measurement of the test plate gave only frequencies and not mode shapes. The FEM surrogate plate model gives both frequencies and mode shapes. The mode shapes of the test plate probably agree with the calculated surrogate plate mode shapes since the frequencies agree nearly

\begin{tabular}{|c|c|c|c|}
\hline \multirow{2}{*}{ Position } & Measured & FEM surrogate material & \multirow{2}{*}{$\begin{array}{c}\text { FEM surrogate material } \\
\text { mode shape }\end{array}$} \\
\hline & frequency $(\mathrm{Hz})$ & plate frequency $(\mathrm{Hz})$ & \\
\hline upper part & 169 & 170 & torsion at edge, Figure 6(c) \\
\hline middle & 325 & 344 & maximal motion, Figure 7 (c) \\
\hline lower part & 169 & 170 & torsion at edge \\
\hline
\end{tabular}

FEM results are shown in Figure 6 and 7 . The number of elements was $8 \cdot 13=104$. The element was square with sides $0.1 \mathrm{~m}$. The plate width was $L=10 \cdot 0.1=1 \mathrm{~m}$ and height $b=8 \cdot 0.1=0.8 \mathrm{~m}$. The element type was quadrilateral parabolic plate. For purposes of illustration the plate thickness is also shown giving appearance of blocks. The visible gaps are only apparent but not actual. The justification for in using a surrogate FEM model instead of accurate FEM models is a trade-off task. The surrogate model gives reasonable predictions with less resource input. 


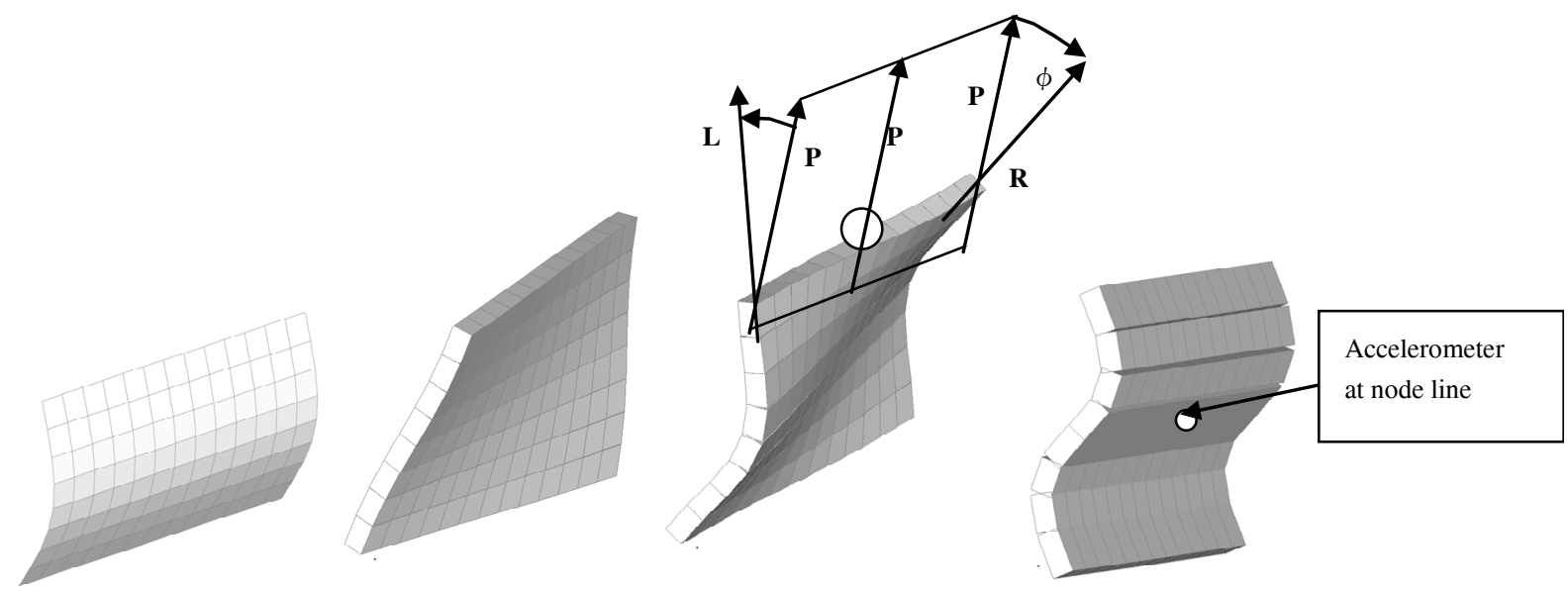

Figure 6. Results of FEM modal analysis of the surrogate orthotropic plate model with the same side lengths as the test plate $(L=a=1.314 \mathrm{~m}, b=0.803 \mathrm{~m})$. The eigenfrequencies $(\mathrm{Hz})$ and mode shapes are shown: a) $f_{1}=67$ plate model with no thickness illustration; b) $f_{2}=80$; c) $f_{3}=170.7$; d) $f_{4}=181$

In Figure 6(c) an illustrative sketch is shown to visualize the torsional modal deformation experienced by the upper part of the beam using vector geometry. Here $\mathrm{P}$ is a unit vector which is drawn tangential to the mid position of the accelerometer. Then $\mathrm{P}$ is shifted to the left and right edges. There its direction is compared with the tangential unit vectors along the edges. Due to symmetry the same angle $\phi$ occurs at both edges. The angle is obtained from $\cos \phi=\mathbf{L} \bullet \mathbf{P}=\mathbf{R} \bullet \mathbf{P}$. In the case of the accelerometer location shown in Figure 6(d) the mode $f_{4}$ is not detected due to small motion since is at the node line of no motion.

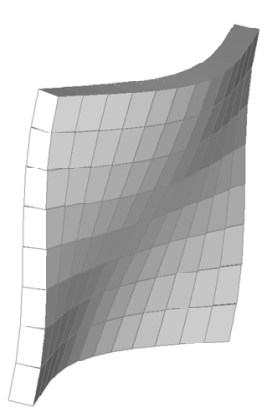

(a) $f_{5}=244 \mathrm{~Hz}$

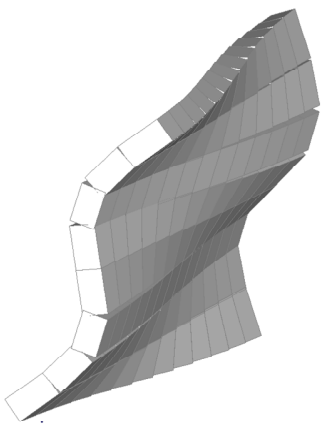

(b) $\quad f_{6}=283 \mathrm{~Hz}$

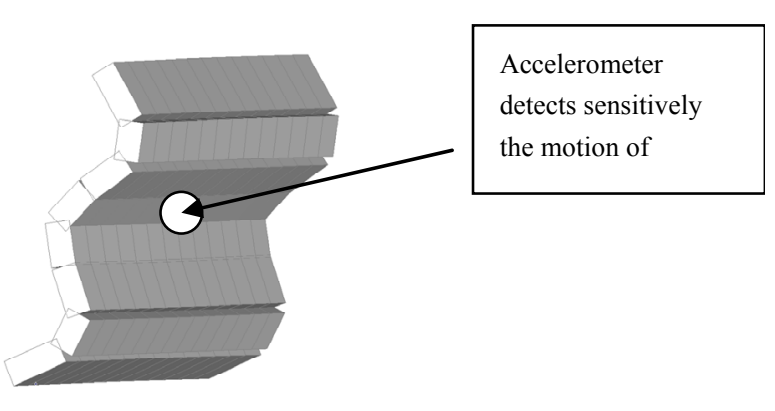

(c) $f_{7}=344 \mathrm{~Hz}$

Figure 7. Results of FEM modal analysis of the surrogate orthotropic plate model with the same side lengths as the test plate $(\mathrm{L}=a=1.314 \mathrm{~m}, b=0.803 \mathrm{~m})$. The eigenfrequencies $(\mathrm{Hz})$ and mode shapes are shown:

$$
\text { a) } f_{5}=244 \text {; b) } f_{6}=283 \text {; c) } f_{7}=344
$$

\subsubsection{FEM Results of the Test Plate with Free Supports on all Sides}

Using FEM modal analysis the shapes and eigenfrequencies shown in Figures 8 and 9 are obtained.

The analytical plate model with free support gave $f_{1, \text { anal }}=118 \mathrm{~Hz}$.

The FEM plate model with the surrogate properties gave $f_{1, \mathrm{FEM}}=114 \mathrm{~Hz}$

FEM results are shown in Figures 8 and 9. 


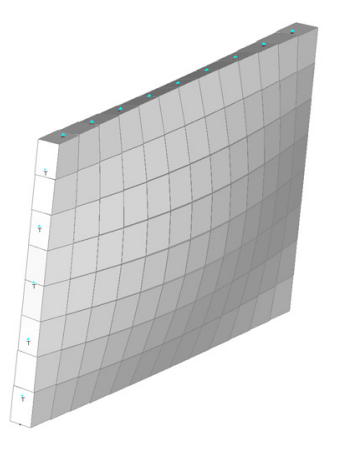

(a) $\quad f_{1}=114 \mathrm{~Hz}$

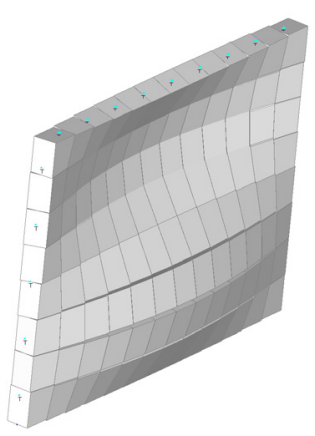

(b)

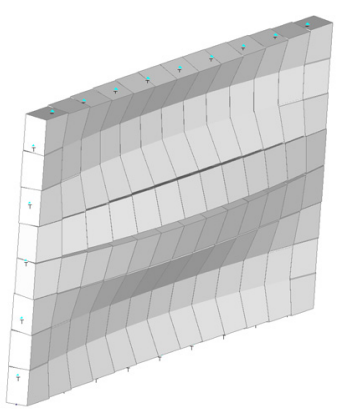

(c) $f_{3}=345 \mathrm{~Hz}$

Figure 8. Results of FEM modal analysis of the surrogate orthotropic plate model with the same side lengths as the test plate $(L=a=1.314 \mathrm{~m}, b=0.803 \mathrm{~m})$. Now all edges are freely supported. The eigenfrequencies $(\mathrm{Hz})$ and mode shapes are shown: a) $f_{1}=114$ (Analytic model gave $118 \mathrm{~Hz}$ ); b) $f_{2}=200$; c) $f_{3}=345$

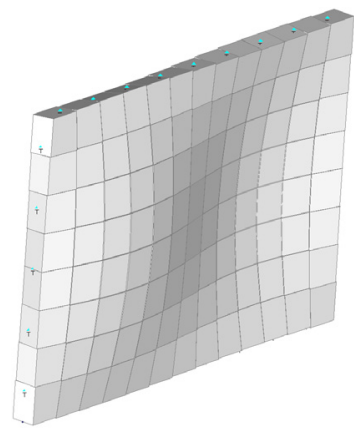

(a) $f_{4}=365 \mathrm{~Hz}$

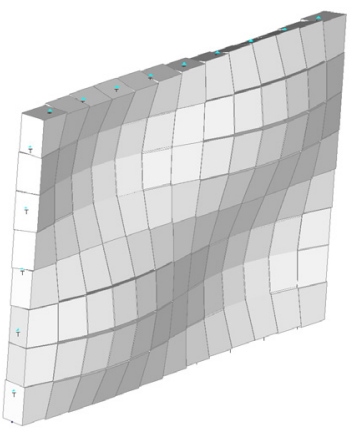

(b) $f_{5}=434 \mathrm{~Hz}$

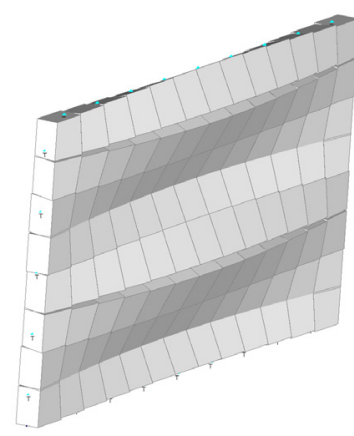

(c) $f_{6}=548 \mathrm{~Hz}$

Figure 9. Results of FEM modal analysis of the surrogate orthotropic plate model with the same side lengths as the test plate ( $L=a=1.314 \mathrm{~m}, b=0.803 \mathrm{~m})$. Now all edges are freely supported. The eigenfrequencies $(\mathrm{Hz})$ and mode shapes are shown: a) $f_{4}=365$; b) $f_{5}=434$; c) $f_{6}=548$

\subsection{Effect of Different Support Conditions on the Eigenfrequencies of Plates and Beams}

A conversion factor $X$ can be used to estimate frequencies depending on the support conditions and model. For beams the eigenfrequency is proportional to $\beta^{2}$.

$$
\frac{f_{1, \text { exp }, \text { plate,free-free }}}{f_{1, \text { sur }, \text { plate, frees-frees, } A N A L}}=\frac{170 \mathrm{~Hz}}{118 \mathrm{~Hz}}=1.44=X_{\text {plate }}, \frac{f_{1, \text { beam, free-free }}}{f_{1, \text { beam, free.sup } p \text {.free.sup } p}}=\frac{(\beta l)_{1, f-f}^{2}}{(\beta l)^{2} f-f s}=\frac{4.73^{2}}{\pi^{2}}=2=X_{\text {beam }}
$$

In the case of the test plate the FEM surrogate model and the analytical orthotropic plate gave nearly the same lowest frequency when the boundary conditions were the same free support on all edges. This result supports the validity of the present modeling approach

\section{Conclusions}

$$
\frac{f_{1, \text { sur }, \text { plate, frees-free }, F E M}}{f_{1, \text { sur }, \text { plate, freeS-free }, A N A L}}=\frac{114 \mathrm{~Hz}}{118 \mathrm{~Hz}}
$$

Orthotropic plates and shell structures are used increasingly and profitably in technical constructions. Some application benefits are low weight, high strength and high stiffness. These are the desired properties in high strength composites. In recovery boilers the heat transfer plates are orthotropic made of parallel tubes. This structure is multifunctional with many desired properties. It takes in heat from hot gas and transfers it by steam flow to electricity generation. The dynamics behavior and endurance are very important for the pulp and paper making industry. If some of the tubes fractures then the boiler may explode or be have to be run down for repair.

(a) FEM modeling of orthotropic plates can be made cost effectively using the surrogate plate approach. 
- In this case these structures are large with many geometrical details. Thus use of detailed FEM modeling is not cost effective.

(b) The measured eigenfrequencies and the surrogate plate model eigenfrequencies agreed satisfactorily. This validates the methodology used. The same free-free conditions were applied for both.

- Accelerometer at edge positions gave $169 \mathrm{~Hz}$.

- Surrogate FEM plate model gave $170 \mathrm{~Hz}$ associated with torsion mode motions at the edges.

- Accelerometer at mid point gave $325 \mathrm{~Hz}$.

- Surrogate FEM plate model gave $344 \mathrm{~Hz}$ at one mode shape having a bulge form and prominent motion at the middle line.

(c) Two models for the fully freely supported plate with the test plate main dimensions gave similar results.

- FEM surrogate plate model gave $114 \mathrm{~Hz}$.

- Analytic orthotropic plate model gave $118 \mathrm{~Hz}$.

(d) One additional advantage of the surrogate model is that it can be used to give estimates for stresses.

- These surrogate stresses can be converted to true stress obtained by an accurate FEM model, using a conversion factor.

- The surrogate modeling is cost effective in large models where detailed models are expensive.

(e) Reasonable models for predicting the soot deposition and removal are obtained in this project.

- These models can be used to design control procedures to minimize soot pluggage and to prevent tube damage. The results are published in the future.

\section{References}

Agarwal, B. D., \& Broutman, L. J. (1990). Analysis and performance of fibre composites. John Wiley \& Sons, Inc.

Barbero, E. J. (1999). Introduction to composite materials design. Taylor \& Francis.

Beitz, W., \& Kuttner, K.-H. (1994, Eds.). Dubbel Handbook of Mechanical Engineering. Springer Verlag

Blevins, R. D. (1990). Flow-induced Vibrations. Van Nostrand Reinhold.

Boresi, A., Schmidt, R., \& Sidebottom, O. (1993). Advanced mechanics of materials. John Wiley.

Case, J., Lord Chilver, C., \& Ross, C. F. T. (1993). Strength of materials and structures with an introduction to finite element methods. Edward Arnold.

Dhillon, B. S., \& Singh, C. (1981). Engineering reliability. Wiley-Interscience.

Diaz, A. (1988). Goal aggregation in design optimization. Engineering Optimization, 13, 257-273. http://dx.doi.org/10.1080/03052158808940958

Dimarogonas, A. D., \& Haddad, S. (1992). Vibration for Engineers. Prentice Hall.

Gurney, T. R. (1978). An analysis of some crack propagation data for steel subjected to pulsating tension loading. The Welding Institute 59/1978/E

Jameel, M. I., Araned, H., \& Luks, G. (1998). Extending recovery boiler runtime through the temperation of steam at the soot blower. TAPPI Engineering Conference.

Leitch, R. D. (1988). Basic reliability engineering analysis. Butterworths, London.

Martikka, H, Pöllänen, I., \& Simonen, J. (2006). Design of optimally safe recovery boilers against occurrence and consequences of internal explosions. SUSI 2006, pp.227-236.

Martikka, H., \& Pöllänen, I. (2009). Multi-objective optimisation by technical laws and heuristics. Memetic computing. http://dx.doi.org/10.1007/s12293-009-0020-0

Martikka, H., \& Taitokari, E. (2013). Bridge concept design using heuristic fuzzy optimum design and FEM. Mechanical Engineering Research, 3(1), 44-67. http://dx.doi.org/10.5539/mer.v3n1p44

Meyer, J. A. (1985). Finite life under combined stress, Machine Design, August, 83-84.

Swanson, S. R. (1997). Introduction to design and analysis with advanced composite materials. New Jersey, Philadephia: Prentice Hall. 
Szilard, R. (1975). Theory and Analysis of Plates. New Jersey, Philadephia: Prentice-Hall.

Talvio, T. (2009). Testing of plates. KyAMK Osk.

Ventsel, E., \& Krauthammer, T. (2001). Thin Plates and shells. New York, NY: Marcel Dekker. http://dx.doi.org/10.1201/9780203908723

Ylinen, A. (1970). Kimmo-ja lujuusoppi II (Theory of Elasticity), WSOY.

\section{Appendix 1. Stresses Obtained by the Surrogate and FEM Model and Fatigue Dimensioning}

One goal of surrogate modeling was to get reasonably accurate dynamic behavior predictions at the lowest eigenfrequancies. The second goal is to convert the surrogate stresses to approximate the true stress obtained by an accurate FEM model using a conversion factor.

First a short review of beam basic modeling is presented. Long plate is like a long beam.

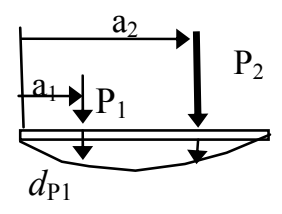

(a)

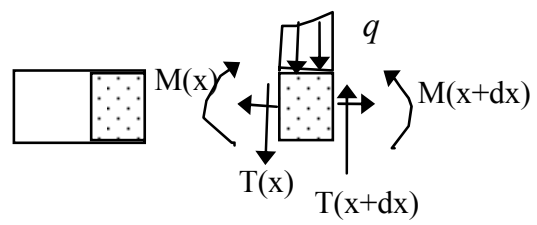

(b)

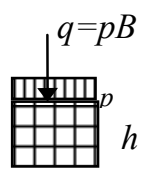

$B$

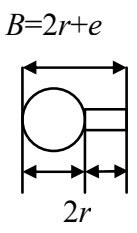

(c)

Figure A1-1: a) Deck model; b) Beam model and free body model; c) Steel unit cross section

First the free body balance of beam elements gives the internal force resultants $N, T$ and $M$ depending on the line load $q, q=p B, B$ is width of the beam and $p$ is pressure on it

Loads on the beam are calculated using equations, (Case, 1999)

$$
\frac{d N}{d x}=0 \quad, \quad \frac{d T}{d x}=-q \quad, \quad \frac{d M}{d x}=T
$$

The sign assumptions are that positive forces act down.

Now the same bending stiffness is assumed in axial (1) direction. The width dimension is $B$.

$$
(D B)_{\text {surrogate }}=(D B)_{\text {steel }}
$$

From this one obtains

$$
D_{11} B=E I_{\text {steel }}=E I_{\text {y,tube }}+E I_{\text {side-plate }} \quad, \quad D_{11}=\frac{1}{12} E_{1} h^{3}
$$

The steel model bending stiffness for axial (1) direction is

$$
E I_{\text {steel }}=E \cdot\left(\pi r^{3} t+\frac{e c^{3}}{12}\right)
$$

A. The steel model actual bending stress

$$
\sigma_{\text {bendsstel }}=\frac{M}{I} z=M \frac{\frac{1}{2} D}{I_{\text {steel }}}=M \frac{\frac{1}{2} 2 r}{\pi r^{3} t+\frac{e c^{3}}{12}} \approx \frac{M}{W}=\frac{M}{\pi r^{2} t}
$$

B. The surrogate model bending stress

$$
\sigma_{\text {surr,bend }}=\frac{M}{I_{\text {surr }}} z=M \frac{E_{1}}{\Sigma E I} z=M \frac{E_{1}}{D_{11} B} \frac{h}{2}=M \frac{1}{\frac{1}{6} h^{2}}
$$

Using these stress models the conversion factors between stresses may be derived. The same moment $M$ is applied first in direction (1) and then in direction (2). 
The ratio of bending stresses is in direction 1 becomes using the dimensions in Figure 3 and $\mathrm{h}=65 \mathrm{~mm}$

$$
\frac{\sigma_{\text {bend,stel }}}{\sigma_{\text {sur, }, \text { end }}}=\frac{M \frac{r}{I_{\text {steel }}}}{M \frac{1}{\frac{h^{2}}{6} B}}=\frac{h^{2} B r}{6 I_{\text {steel }}}=\frac{1}{6 \pi}\left(\frac{h}{r}\right)^{2}\left(\frac{2 r+e}{t}\right)=R_{\text {bend, } 1} \approx 0.34
$$

Ratio of bending stresses in transverse (2) direction

$$
R_{\text {bend }, 2}(h)=\frac{\sigma_{\text {tens,steel, } 2}}{\sigma_{\text {tens.sur }, 2}}=\frac{M \frac{1}{L \frac{1}{6} c^{2}}}{M \frac{1}{L \frac{1}{6} h^{2}}}=\left(\frac{h}{c}\right)^{2} \Rightarrow\left(\frac{65}{6}\right)^{2}=121
$$

\section{Fatigue Design}

There are several methods for fatigue dimensioning. One method is to use fatigue diagrams based on assumption that fatigue cracks have not yet initiated. One simple and conservative diagram is by (Meyer, 1985). Many others can be used.

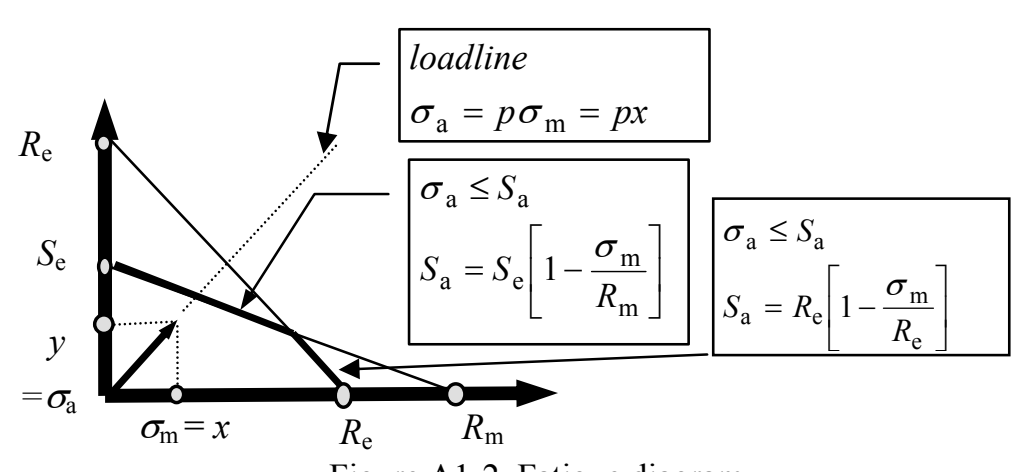

Figure A1-2. Fatigue diagram

The mean stress is due to self weight is of a heat transfer plate of length $L=23 \mathrm{~m}$ is.

$$
\sigma_{m}=\rho g L
$$

The stress amplitude is the maximum bending stress

$$
\sigma_{a}=\frac{M}{I} z=\frac{E I w_{x x}}{I} z=E w_{x x} z=E \frac{r}{R} \approx E \frac{0.05 m}{100 m} \approx 100 M P a, \quad p=\frac{\sigma_{\mathrm{a}}}{\sigma_{\mathrm{m}}}>1
$$

Thus the stress ratio $p$ is large and the bending stress is the dominant stress.

The endurance condition may be expressed using the margin of safety

$$
R(1)=S_{\mathrm{a}}-\sigma_{a} \geq 0 \rightarrow \quad, \quad S_{e}=C \cdot 0.5 R_{\mathrm{m}}=0.4 \cdot 0.5 \cdot 400=40 \mathrm{MPa}
$$

Dynamic simulation gave the stress amplitude as $30 \mathrm{MPa}$.

$$
S_{\mathrm{a}}=\frac{1}{N}\left[\frac{p S_{e}}{\frac{S_{e}}{R_{\mathrm{m}}}+p}\right] \approx \frac{1}{N} S_{e} \geq \sigma_{a} \Rightarrow \frac{1}{N} 40>30 \Rightarrow \max \cdot N=1.33
$$

\section{Copyrights}

Copyright for this article is retained by the author(s), with first publication rights granted to the journal.

This is an open-access article distributed under the terms and conditions of the Creative Commons Attribution license (http://creativecommons.org/licenses/by/3.0/). 